\title{
First-Line Immune Checkpoint Inhibitor- Based Sequential Therapies for Advanced Hepatocellular Carcinoma: Rationale for Future Trials
}

\author{
Giuseppe Cabibbo ${ }^{a}$ Maria Reig ${ }^{b}$ Ciro Celsa ${ }^{a, c}$ Ferran Torres d, e \\ Salvatore Battaglia ${ }^{f}$ Marco Eneag Giacomo Emanuele Maria Rizzo ${ }^{a}$ \\ Salvatore Petta ${ }^{a}$ Vincenza Calvaruso ${ }^{a}$ Vito Di Marco ${ }^{a}$ Antonio Craxì ${ }^{a}$ \\ Amit G. Singal $^{\mathrm{h}} \quad$ Jordi Bruix ${ }^{\mathrm{b}}$ Calogero Cammà ${ }^{\mathrm{a}}$ \\ aSection of Gastroenterology \& Hepatology, Department of Health Promotion, Mother and Child Care, Internal

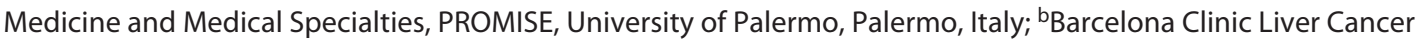 \\ (BCLC) Group, Liver Unit, Hospital Clínic de Barcelona, IDIBAPS, Universidad de Barcelona, CIBEREHD, Hospital Clinic \\ de Barcelona, Barcelona, Spain; 'Department of Surgical, Oncological and Oral Sciences (Di.Chir.On.S.), University

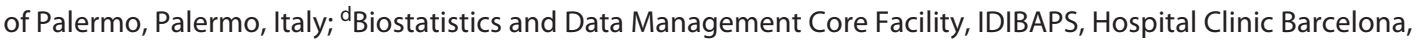 \\ Barcelona, Spain; 'Biostatistics Unit, Faculty of Medicine, Universitat Autònoma de Barcelona, Barcelona, Spain; \\ fDipartimento di Scienze Economiche, Aziendali e Statistiche, University of Palermo, Palermo, Italy; ${ }^{\text {DDepartment }}$ \\ of Health Promotion, Mother and Child Care, Internal Medicine and Medical Specialties, PROMISE, University of \\ Palermo, Palermo, Italy; hivision of Digestive and Liver Diseases, UT Southwestern Medical Center, Dallas, TX, USA
}

\section{Keywords}

Hepatocellular carcinoma · Sequential treatment ·

Immunotherapy

\begin{abstract}
Introduction: Atezolizumab (ATEZO) plus bevacizumab (BEVA) represents the new standard of care for the treatment of advanced hepatocellular carcinoma (HCC). However, the choice of the second-line treatment after the failure of immunotherapy-based first-line remains elusive. Taking into account the weaknesses of the available evidence, we developed a simulation model based on available phase III randomized clinical trials (RCTs) to identify optimal risk/benefit sequential strategies. Methods: A Markov model was built to estimate the overall survival (OS) of sequential first- and sec-
\end{abstract}

karger@karger.com www.karger.com/lic

Karger $\stackrel{\text { ' }}{=}$

BOPEN ACCESS
(C) 2021 The Author(s)

Published by S. Karger AG, Basel

This is an Open Access article licensed under the Creative Commons Attribution-NonCommercial-4.0 International License (CC BY-NC) (http://www.karger.com/Services/OpenAccessLicense), applicable to the online version of the article only. Usage and distribution for commercial purposes requires written permission. ond-line systemic treatments. Sequences starting with firstline ATEZO plus BEVA followed by 5 second-line treatments (sorafenib [SORA], lenvatinib [LENVA], regorafenib, cabozantinib, and ramucirumab) were compared. The probability of transition between states (initial treatment, cancer progression, and death) was derived from RCTs. Life-year gained (LYG) was the main outcome. Rates of severe adverse events (SAEs) ( $\geq$ grade 3 ) were calculated. The incremental safetyeffectiveness ratio (ISER) was calculated as the difference in probability of SAEs divided by LYG between the 2 most effective sequences. Results: ATEZO plus BEVA followed by LENVA (median OS, 24 months) or SORA (median OS, 23 months) was the most effective sequence, producing a LYG of 0.50 and 0.42 year, respectively. ATEZO plus BEVA fol-

Giuseppe Cabibbo and Maria Reig equally contributed to this work.
Correspondence to:

Jordi Bruix, jbruix@ clinic.cat

Calogero Cammà, calogero.camma@unipa.it 
lowed by SORA was the safest sequence (SAEs 63\%). At a willingness-to-risk threshold of $10 \%$ of SAEs for LYG, ATEZO plus BEVA followed by second-line SORA was favored in $72 \%$ of cases, while at a threshold of $30 \%$ of SAEs for LYG, ATEZO plus BEVA followed by second-line LENVA was favored in $69 \%$ of cases. Conclusion: Our simulation model provides a strong rationale to support ongoing trials evaluating second-line tyrosine-kinase inhibitors after first-line ATEZO plus BEVA. Future evidence from ongoing RCTs and prospective real-world studies are needed to prove the net health benefit of sequential treatment options for advanced HCC.

(C) 2021 The Author(s).

Published by S. Karger AG, Basel

\section{Introduction}

Hepatocellular carcinoma (HCC) is the fourth-leading cause of cancer deaths worldwide [1]. Curative treatment options, such as liver transplantation, resection or ablation, exist for Barcelona Clinic Liver Cancer (BCLC) stage 0/A (early-stage) HCC, while patients with locally advanced disease may be candidates for liver-directed therapies, including transarterial chemoembolization and transarterial radioembolization [2]. Unfortunately, more than half of all HCC cases are diagnosed at a stage with no potentially curative treatment options. Since 2008, the oral tyrosine-kinase inhibitor (TKI) Sorafenib (SORA), has been recommended as the standard first-line systemic therapy for patients with BCLC stage $\mathrm{C}$ (advanced) $\mathrm{HCC}$ and those with BCLC stage B (intermediate) $\mathrm{HCC}$ who are unfit for, or fail to respond to, loco-regional therapies [3].

Recently, several newer systemic therapy options have shown efficacy in the first- and second-line settings. In the first-line setting, combination therapy with atezolizumab (ATEZO) plus bevacizumab (BEVA) has exhibited an impressive improvement in overall survival (OS) and progression-free survival (PFS) compared with SORA and it now represents the standard of care [4]. In the second-line setting, multiple TKIs have shown survival benefit when compared with placebo [5-7], although a definitive standard of care has not yet been defined [8].

Taken individually, these randomized controlled trials (RCTs) offer a number of effective choices for first- and second-line systemic treatments. Ideally, they should be combined in a rational sequence to offer the best net health benefit for patients with advanced HCC. However, RCTs were designed to maximize the effectiveness of each new drug under evaluation, and the best sequential sys- temic treatment remains debated [8-10]. Unfortunately, sequential trials in oncology are difficult to perform and real-world data of sequential therapies for advanced HCC are lacking to date. Moreover, all approved second-line therapies in HCC have been assessed in patients who received SORA as first-line treatment, and data on secondline treatment after first-line ATEZO + BEVA are lacking. Nevertheless, the American Society of Clinical Oncology (ASCO) and the European Society for Medical Oncology (ESMO) guideline recommendations recently suggested the use of SORA or lenvatinib (LENVA) as second-line treatment options after ATEZO + BEVA failure $[9,10]$. Given the lack of direct comparative effectiveness data for sequential therapy options, we developed a simulation model based on published phase 3 RCTs to identify the optimal strategy for sequencing systemic agents after first-line immune checkpoint inhibitor (ICI)-based combination treatments.

\section{Methods}

\section{Trial Selection}

Data from phase 3 RCTs of systemic agents with proven survival benefit were extracted. For ATEZO + BEVA, data were extracted from the IMbrave 150 trial [4]. Data were extracted from 8 phase III RCTs $[3,4,11-16]$ for SORA and pooled by a randomeffects model. Data were extracted from REFLECT [16], RESORCE [5], and CELESTIAL [6] trials for LENVA, Regorafenib (REGO), and Cabozantinib (CABO), respectively. For Ramucirumab (RAMU), data were extracted from a pooled meta-analysis of independent patient data [17] from REACH [18] and REACH-2 [7] trials. Characteristics of the included RCTs are showed in online supplementary Tables 1 and 2 (for all online suppl. material, see www.karger.com/doi/10.1159/000520278).

\section{Meta-Regression Analysis}

To examine the extent to which differences in OS with secondline TKI therapy (including SORA and LENVA) could be explained by differences in study- and patient-level covariates, the following explanatory variables were included in a meta-regression model: year of study publication (only for SORA trials), type of drug, sex, ethnicity, Eastern Cooperative Oncology Group (ECOG) Performance Status, etiology of liver disease, Child-Pugh class, Albumin-Bilirubin (ALBI) grade, BCLC stage, alpha-fetoprotein (AFP), macrovascular invasion and extrahepatic spread. Data on ALBI grade were extracted from post hoc exploratory analyses when available [19-21]. Other variables of interest, including degree of liver involvement and degree of portal vein invasion were not uniformly reported across trials, so could not be included in meta-regression analysis. Median OS times were used as the effect size, weighted by standard errors. In order to reduce the risk of ecological bias, only univariate meta-regression was performed. Restricted maximum likelihood was used for the model estimation ( $\mathrm{R}$ package nlme). Data used in the meta-regression model are reported in online supplementary Table 2.
Cabibbo et al. 


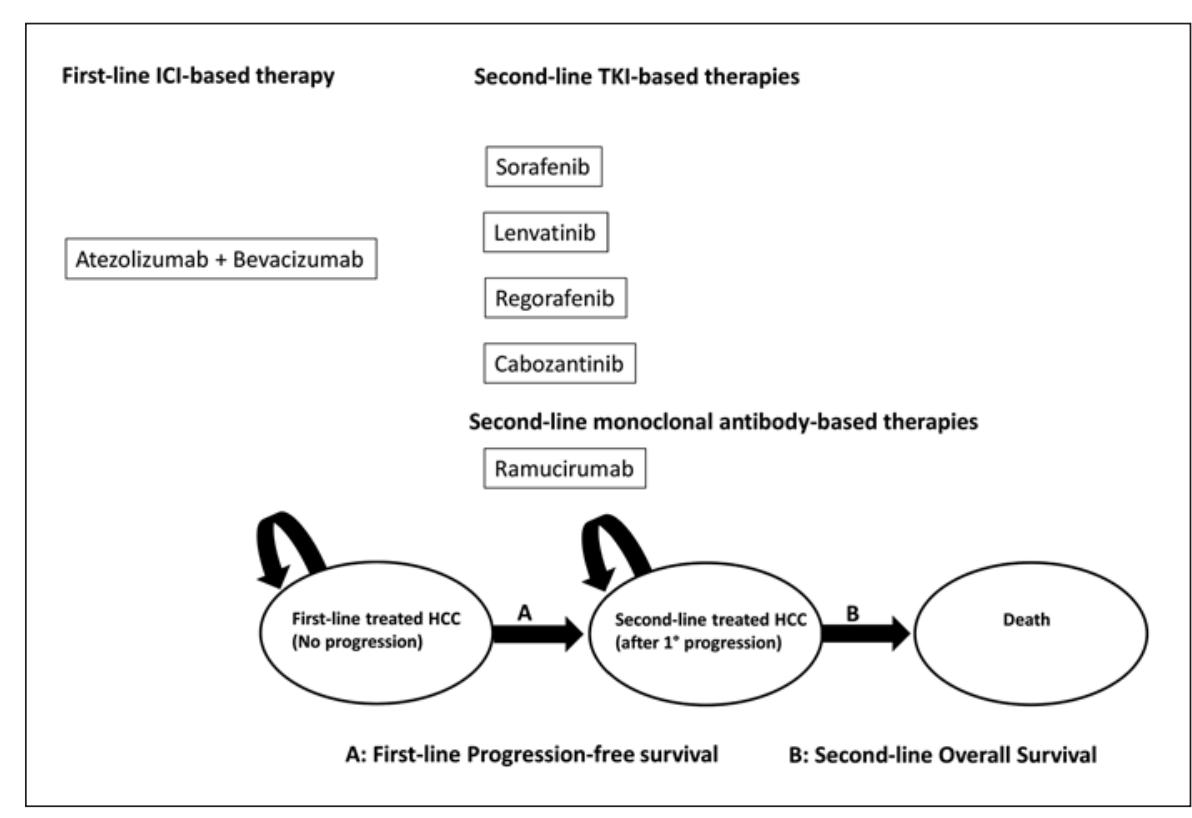

Fig. 1. General structure of the Markov model. TKI, tyrosine-kinase inhibitor; ICI, immune checkpoint inhibitor; PFS, progression-free survival; OS, overall survival.

\section{Simulation Model}

A semi-Markov model was developed to analyze the effectiveness of different sequential systemic treatments for advanced HCC over a lifetime horizon (Fig. 1). The model allowed the transition hazard to increase over time and it was performed by estimating the scale and shape parameter of a Weibull distribution. Natural mortality rate at age of population was included in the model for a 65 -year-old hypothetical male patient. Given the very short life expectancy, we did not discount risks and benefits. The sequential semi-Markov model was built by using an ad hoc routine written with $\mathrm{R}$ statistical software. We simulated the clinical course of a hypothetical patient cohort with advanced HCC and similar characteristics as those enrolled in included phase 3 RCTs (online supplementary Table 1).

Data describing OS and PFS treatment benefits were derived from Kaplan-Meier curves from the above-mentioned trials. Specifically, we extracted data from the PFS curve of the IMbrave 150 trial [4] for first-line ATEZO + BEVA. PFS was assumed as the main endpoint for first-line ATEZO + BEVA, because progression is the event leading to treatment discontinuation and transition to second-line therapy [22]. OS curves of patients treated with second-line therapies were extracted from all 8 phase III RCTs [3, 4, 11-16] for SORA and from REFLECT [16], RESORCE [5], and CELESTIAL [6], for LENVA, REGO, and CABO, respectively. For RAMU, data were extracted from a pooled meta-analysis of independent patient data [17] including patients with AFP higher than $400 \mathrm{ng} / \mathrm{mL}$ from REACH and REACH-2 trials [7, 18].

Individual patient survival data were reconstructed by using an algorithm proposed by Guyot et al. [23]. This algorithm provides a list of patients with predicted survival times and a predicted event of interest (i.e., alive or dead; progression or no progression) by using digitalized data on survival probabilities, time, and total number of patients and events. Engauge Digitizer software Version 12 was used to extract data from the curves. Each reconstructed survival curve was inspected for accuracy and was compared with originally published curves. For safety measures, we extracted the number of grade $\geq 3$ adverse events (severe adverse events, SAEs) from each included trial.

\section{Model Transitions and Survival Estimates}

The model simulated transitions among the following 3 health states, with a cycle length of 1 month: (1) advanced HCC eligible for first-line systemic therapy; (2) first progression; and (3) death (Fig. 1). The health states were mutually exclusive (i.e., a patient could only experience a single health state at any given time). After first-line therapy, patients could experience a response and continue first-line ICI-based therapy, experience progression, and switch to second-line treatment, or death. Patients on second-line therapy could experience treatment response or death. Therefore, patients remained on second-line therapy if they experienced second progression.

Survival estimates for sequential settings considered the proportion of patients who did not receive second-line therapy due to death. Transitions between health states were based on calculated transition probabilities from PFS and OS data extracted from published studies. Simulation of 2000 pseudo-random patients was performed to obtain overall simulated survival times and median times throughout the 3 states of the disease.

\section{Outcomes}

Life-year gained (LYG) of the sequential therapies was the main health outcome. We calculated median OS of sequential therapies from the Markov model. To provide long-term survival information, a milestone survival analysis at 24 months was performed. Accordingly, the number needed to treat (NNT) was calculated for each sequential strategy.

The rate of SAEs was calculated for each sequential treatment, taking into account transition probabilities from first- to secondline treatments, by using a weighted mean of the number of patients transited in each disease state (initial treatment and cancer progression). To obtain a net health benefit measure [24], clinical benefit and safety were combined to calculate an innovative mea- 
Table 1. Clinical features and outcomes reported in the clinical trials examined and model parameters

\begin{tabular}{|c|c|c|}
\hline Variables & Base-case & References \\
\hline \multicolumn{3}{|l|}{ First-line atezolizumab plus bevacizumab } \\
\hline Characteristics of patients & & 4 \\
\hline Median age, yr & 64 & \\
\hline Male sex, $\%$ & 82.4 & \\
\hline Asian ethnicity, \% & 39.6 & \\
\hline $\mathrm{HBV}, \%$ & 48.8 & \\
\hline $\mathrm{HCV}, \%$ & 21.4 & \\
\hline Vascular invasion and/or extrahepatic disease, $\%$ & 76.8 & \\
\hline ECOG performance status $1, \%$ & 37.7 & \\
\hline $\mathrm{BCLC} C, \%$ & 82.1 & \\
\hline Median PFS* (months) reported in trial & $6.8 \mathrm{mo}$ & 4 \\
\hline Weibull distribution parameters (scale; shape) & $9.78 ; 1.2$ & - \\
\hline Median PFS (IQR) (months) obtained with Weibull distribution & $7.2 \mathrm{mo}(9.6)$ & - \\
\hline $\begin{array}{l}\text { Mean PFS (SD) (months) obtained with Weibull distribution } \\
\text { SAEs, \% }\end{array}$ & $9.25 \mathrm{mo}(0.18)$ & - \\
\hline Atezolizumab plus bevacizumab & 61 & 4 \\
\hline \multicolumn{3}{|l|}{ Second-line systemic treatments } \\
\hline \multicolumn{3}{|l|}{ Median OS (months) reported in RCTs, mo } \\
\hline Sorafenib before 2018 & 9.65 & $3,11-15$ \\
\hline Sorafenib after 2018 & 12.5 & 4,16 \\
\hline Lenvatinib & 13.6 & 16 \\
\hline Regorafenib & 10.6 & 5 \\
\hline Cabozantinib & 10.2 & 6 \\
\hline Ramucirumab & 8.1 & 17 \\
\hline \multicolumn{3}{|l|}{ Weibull distribution parameters (scale; shape) } \\
\hline Sorafenib before 2018 & $14.21 ; 1.23$ & \\
\hline Sorafenib after 2018 & $20.98 ; 1.07$ & \\
\hline Lenvatinib & $20.86 ; 1.26$ & \\
\hline Regorafenib & $15.36 ; 1.23$ & \\
\hline Cabozantinib & $15.24 ; 1.27$ & \\
\hline Ramucirumab & $11.6 ; 1.27$ & \\
\hline \multicolumn{3}{|l|}{ Median OS (IQR) (months) obtained with Weibull distribution, mo } \\
\hline Sorafenib before 2018 & $11(13)$ & \\
\hline Sorafenib after 2018 & $15(22)$ & \\
\hline Lenvatinib & $15.5(19.4)$ & \\
\hline Regorafenib & $11.4(14.4)$ & \\
\hline Cabozantinib & $11.4(14)$ & \\
\hline Ramucirumab & $8.9(10.9)$ & \\
\hline \multicolumn{3}{|l|}{ Mean OS (SD) (months) obtained with Weibull distribution, mo } \\
\hline Sorafenib before 2018 & $13.92(0.35)$ & \\
\hline Sorafenib after 2018 & $20.77(0.48)$ & \\
\hline Lenvatinib & $19.94(0.3)$ & \\
\hline Regorafenib & $14.8(0.62)$ & \\
\hline Cabozantinib & $14.7(0.54)$ & \\
\hline Ramucirumab & $11.3(0.1)$ & \\
\hline \multicolumn{3}{|l|}{ SAEs, $\%$} \\
\hline Sorafenib before 2018 & 77.1 & $3,11-15$ \\
\hline Sorafenib after 2018 & 65.1 & 4,16 \\
\hline Lenvatinib & 75 & 16 \\
\hline Regorafenib & 79.7 & 5 \\
\hline Cabozantinib & 79.4 & 6 \\
\hline Ramucirumab & 70.4 & 18 \\
\hline
\end{tabular}

For sorafenib, OS data were pooled stratifying according the publication year before [3, 11-15] and after 2018 $[4,16]$. OS, overall survival; PFS, progression-free survival; Mo, months; IQR, interquartile range; SD, standard deviation; SAEs, severe adverse events; ECOG, Eastern Cooperative Oncology Group; BCLC, Barcelona clinic liver cancer; RCTs, randomized controlled trials. * Assessed by RECIST 1.1. 
Table 2. Base-case efficacy and safety of treatment sequences with atezolizumab plus bevacizumab as first-line according to the simulated 24-month OS

\begin{tabular}{|c|c|c|c|c|c|}
\hline Treatment sequence & $\begin{array}{l}\text { 24-month } \\
\text { OS, \% }\end{array}$ & $\begin{array}{l}\text { NNT } \\
\text { (patients, } n \text { ) }\end{array}$ & $\begin{array}{l}\text { Median OS, } \\
\text { mo }(95 \% \mathrm{Cl})\end{array}$ & $\begin{array}{l}\text { LYG, } \\
y r\end{array}$ & $\begin{array}{l}\text { SAEs, \% } \\
(95 \% \mathrm{CI})\end{array}$ \\
\hline Atezolizumab plus bevacizumab - Lenvatinib & 49.3 & 5.5 & $24(23-25)$ & 0.50 & $67.8(66.3-69.3)$ \\
\hline Atezolizumab plus bevacizumab - cabozantinib & 37.0 & 16.7 & $20(19-21)$ & 0.17 & $69.9(68.5-71.4)$ \\
\hline Atezolizumab plus bevacizumab - regorafenib & 37.0 & 17 & $20(19-21)$ & 0.17 & $70.0(68.6-71.5)$ \\
\hline Atezolizumab plus bevacizumab - Sorafenib before 2018 & 35.0 & 24.7 & $20(19-21)$ & 0.17 & $68.8(67.3-70.3)$ \\
\hline
\end{tabular}

All the sequences were compared to the worst sequence (atezolizumab plus bevacizumab - ramucirumab) in terms of NNT and LYG. NNT was calculated by using 24-month OS rate. OS, overall survival; LYG, life-year gained; NNT, number needed to treat; SAEs, severe adverse events; Yr, years; Mo, months; 95\% $\mathrm{Cl}, 95 \%$ confidence intervals.

sure, that is the incremental safety-effectiveness ratio (ISER). The latter was defined as the difference in the rate of SAEs between 2 sequential treatments, divided by their difference in effectiveness, measured in LYG. This unit of measure expresses the incremental percentage of SAEs for each LYG.

$$
\text { ISER }=\frac{\text { Delta SAEs } \%}{\text { LYG }} .
$$

\section{Sensitivity Analysis}

Two-way sensitivity analysis for ISER was performed by varying the numerator (percentage difference in SAEs) and the denominator (LYG). The variation range for the denominator was chosen to be as large as the maximal value observed, while a $\pm 5 \%$ variation was chosen for the numerator. Analogous to the willingness-to-pay threshold of a cost-effectiveness analysis, a willingness-to-risk threshold value must be established for ISER. Different willingness-to-risk thresholds for ISER, and their visual effect in terms of estimated areas, across the potential range of SAEs and LYG, have been calculated. All the analyses were performed using $\mathrm{R}$ ( $\mathrm{R}$ core team, 2020).

\section{Results}

Meta-regression analysis showed that SORA OS increased in RCTs published after 2018, so pooled OS of SORA was stratified before and after 2018 (see online suppl. Table 3). SORA after 2018 and LENVA were the only drugs significantly associated with OS improvement compared to SORA before 2018. Pooled OS from the 2 SORA control arms of IMBrave 150 [4] and REFLECT [16] RCTs were included in the Markov model as post2018 trials. Among patient-level covariates, ALBI grade 2 , BCLC C, and AFP $>400 \mathrm{ng} / \mathrm{mL}$ were significantly associated with worse OS.

Relevant outcomes and model parameters from the RCTs are described in Table 1. The base-case efficacy and safety of the treatment sequences after ATEZO + BEVA, ordered according to 24-month OS, are reported in Table 2. First-line ATEZO + BEVA followed by second-line LENVA was the most effective treatment with median OS of 24 months, LYG of 0.50 year, 24-month OS of $49.3 \%$ and NNT of 5. First-line ATEZO + BEVA followed by second-line SORA was the second most effective treatment with median OS of 23 months, LYG of 0.42 year, 24 -month OS of $46.6 \%$, and NNT of 6 . Figure 2 shows the simulated survival curves of ATEZO + BEVA followed by LENVA or SORA.

The safety profile of treatment sequences is reported in Table 2. First-line ATEZO + BEVA followed by second-line LENVA was associated with $67.8 \%$ SAEs, while first-line ATEZO + BEVA followed by SORA was associated with $63.0 \%$ SAEs, resulting the safest sequence. Firstline ATEZO + BEVA followed by second-line LENVAdominated sequential strategies, including REGO or $\mathrm{CABO}$ for both effectiveness and safety.

A two-way sensitivity analysis, varying SAE and LYG values, was performed to evaluate which therapy would be preferred at different willingness-to-risk thresholds. In Figure 3, at a willingness-to-risk threshold of $10 \%$ of SAEs for LYG, first-line ATEZO + BEVA followed by secondline SORA was favored in $72 \%$ of cases, while at a threshold of $30 \%$ of SAEs for LYG, first-line ATEZO + BEVA followed by second-line LENVA was favored in 69\% of cases.

\section{Discussion}

The best sequential systemic treatment for patients with advanced HCC after first-line ATEZO + BEVA failure still remains debated. Recently, ASCO, ESMO, and 


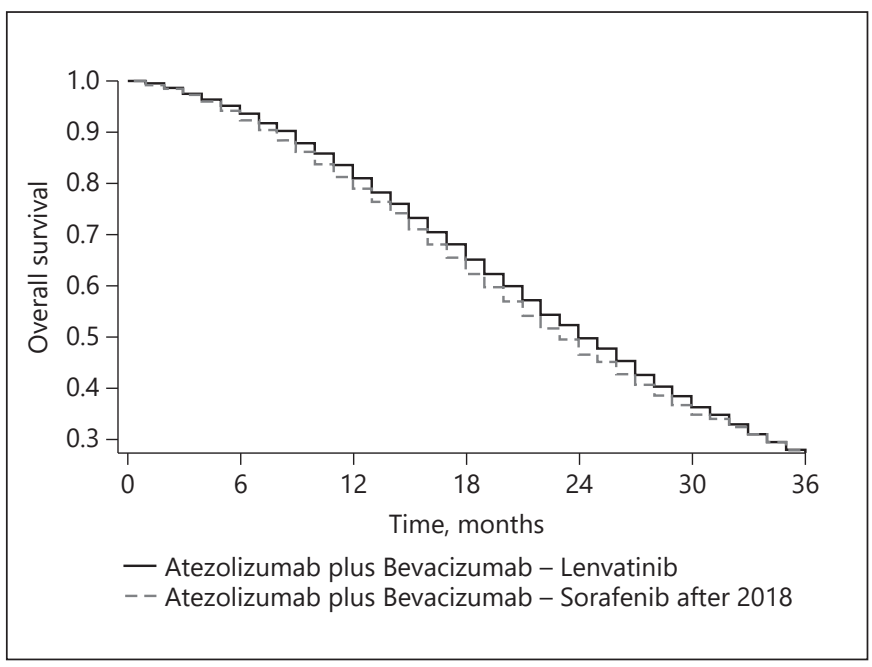

Fig. 2. Simulated survival curves of atezolizumab plus bevacizum$\mathrm{ab}$ followed by lenvatinib or sorafenib in patients with advanced HCC. HCC, hepatocellular carcinoma.

the European Association for the Study of Liver societies suggested the use of any TKI (including SORA and LENVA) as second-line therapies after ATEZO + BEVA [9, $10,25]$. Our analysis provided evidence that SORA and LENVA were the 2 TKIs significantly associated with OS improvement. In this line, our sequential model suggests that first-line ATEZO-BEVA followed by second-line LENVA or SORA could be optimal sequential systemic strategies in terms of efficacy, with a median OS of about 2 years, as well as safety. To the best of our knowledge, this is the first model providing comparisons among different second-line TKIs after first-line ICI-based treatment. Waiting for the validation of the key assumptions of our model by RCTs and large prospective real-world data, our simulation model provides further evidence supporting ASCO, ESMO, and the European Association for the Study of Liver recommendations, suggesting that the sequences with first-line ATEZO + BEVA followed by second-line TKIs (LENVA or SORA) may represent the optimal sequences. In this line, we are awaiting for the upcoming results from the phase III trial of the ICI-TKIbased combination LENVA plus Pembrolizumab [26]. Regarding the safety profile, our study showed that the use of second-line SORA after ICI-based first-line was the best sequence in terms of tolerability. It is important to note that the results of our meta-regression, showing that the benefit of SORA significantly increased over time, are plausible and supported by available real-world and clinical trial data [27-29]. The improvement in the manage- ment of SORA side effects, tailoring SORA dosage to optimize risk/benefit ratio, and finally the availability of second-line treatment options could explain these results.

Sequential trials in oncology are difficult to perform and they are practically unfeasible in most settings, such as advanced HCC. Furthermore, large prospective realworld data of sequential therapies in advanced HCC are time-consuming and lacking to date. Our results address an area of urgent clinical need, in which direct comparative effectiveness data are lacking. Our simulation model could be a useful tool to predict the outcome of different systemic treatment sequences. In this line, simulation models starting from locoregional treatments are needed to provide a more accurate assessment of treatment benefit across the patient-journey.

Simulation models are subject to several limitations, mainly due to lack of individual data and absence of validation on key assumptions. For example, we did not have data regarding degree of liver involvement or portal vein invasion for meta-regression models. Therefore, our sequential model should be considered as a hypothesis generating tool, and real-world data should be constantly generated to confirm all assumptions. In this line, Yoo et al. [30] demonstrated the second-line treatment with LENVA or SORA had comparable efficacy and manageable toxicities in patients with advanced HCC after AT$\mathrm{EZO}+\mathrm{BEVA}$ failure. We believe that simulation models could provide an anticipated evaluation useful to design and power future trials. For instance, our forecasts on the 2 optimal sequences ATEZO + BEVA followed by LENVA or SORA represent the control group of the ongoing trial (IMBrave251, NCT04770896), comparing ATEZO + LENVA versus LENVA alone or ATEZO + SORA versus SORA alone in ATEZO + BEVA failure patients [31]. Moreover, it is important to consider that previously published estimates of sequential strategies are biased by "per-protocol analyses," and they did not consider patients who died before second-line treatment $[32,33]$, with a bias of potential overestimate of the efficacy of the proposed sequences. Finally, a strength of our model was the use of PFS as the main endpoint for first-line treatment, since it is likely that, in real-life, treatment sequencing will be considered upon tumor progression $[22,34$, 35].

Assessment of the benefit of ICIs raises several issues considering the unconventional pattern of radiological response. Novel tools assessing radiological response (i.e., immuneRECIST or radiomic tools) or innovative measures of net health benefit assessment may be able to capture the long-term benefit observed with ICIs
Liver Cancer 2022;11:75-84 DOI: $10.1159 / 000520278$
Cabibbo et al. 


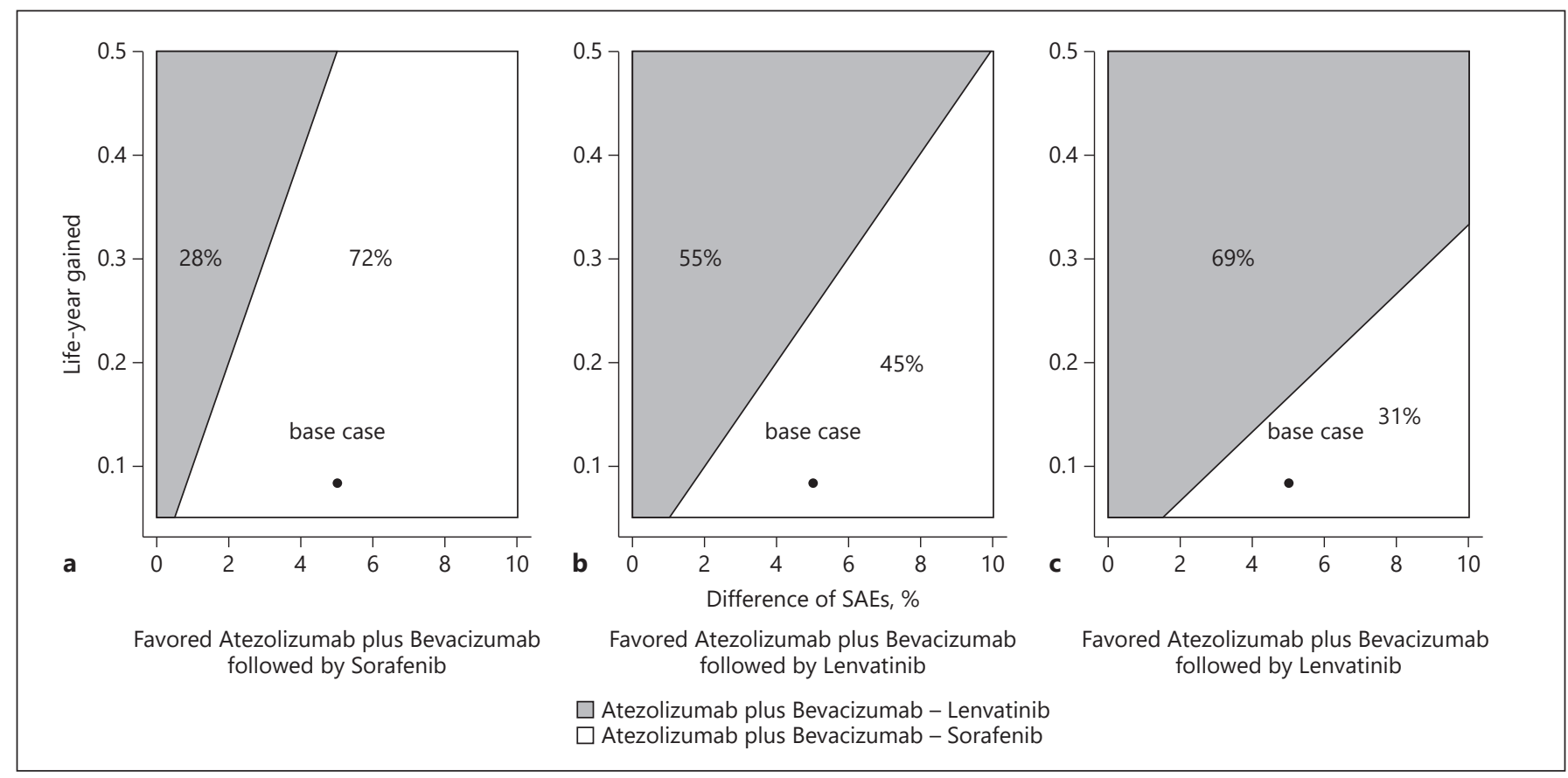

Fig. 3. Two-way sensitivity analysis of incremental safety-effectiveness ratio (ISER, i.e., delta severe adverse events \%/life-year gained) to indicate which sequence is favored according to different "willingness-to-risk" thresholds. Gray area favored atezolizumab plus bevacizumab followed by lenvatinib, while white area favored atezolizumab plus bevacizumab followed by sorafenib.

[36]. According to ASCO statements [24], net health benefit is defined as the balance between clinical benefit and SAEs, and it is used to assess the difference between 2 different therapeutic strategies [37]. In our model, we used an innovative measure combining efficacy and safety, called ISER (incremental safety-effectiveness ratio). Given the range of uncertainty on the net health benefit assessed by ISER between ATEZO-BEVA followed by LENVA or SORA, the best treatment strategy should be carefully shared with the patients and agreed with the policy makers, taking also into account costeffectiveness ratio. In this line, the limited survival benefit observed in RCTs, particularly in the second-line setting, underlines the importance of including both quality of life and the patient-reported outcomes. When we evaluated the net health benefit according to ISER, we observed that ATEZO + BEVA followed by LENVA was clearly favored only in patients with high willingness-to-risk threshold (e.g., fit patients with fighter attitude who dismiss the impact of SAEs), while ATEZO + BEVA followed by SORA was favored in patients with low willingness-to-risk threshold (e.g., frail patients
Three different scenarios were reported: willingness-to-risk threshold of $10 \%$ of SAEs for LYG (a); willingness-to-risk threshold of $20 \%$ of SAEs for LYG (b); willingness-to-risk threshold of $30 \%$ of SAEs for LYG (c). SAEs, severe adverse events; LYG, lifeyear gained.

with fatalist attitude in whom a higher risk of SAEs is relevant). It is to note that TKIs can have continuous low grade (grade 2) toxicity that could not be entirely captured by ISER.

Like all the simulation models, in the present study there are several limitations. First, there is a lack of published data concerning second-line treatments after AT$\mathrm{EZO}+\mathrm{BEVA}$. All available second-line therapies were approved in trials including SORA-experienced patients, while no second-line therapies were evaluated after AT$\mathrm{EZO}+\mathrm{BEVA}$. As a proxy measure of treatment failure, about $20 \%$ of patients in the IMBrave 150 trial [4] received subsequent TKI treatment after ATEZO + BEVA, although the reasons for the transition to second-line therapy were unknown. Second, we were unable to account for some differences in patient inclusion and exclusion criteria across trials. Notably, the REFLECT trial excluded patients with $>50 \%$ liver involvement or main portal vein invasion. The inclusion of a more favorable population may bias results to favor LENVA [16]. Furthermore, some patients with progression after ATEZO + BEVA may not be candidates for second-line LENVA 
if they present the previously mentioned exclusion criteria. Moreover, REFLECT trial also selected a population with a lower risk of developing cardiovascular events during TKI treatments [16]. Third, we incorporated data from RCTs of both first- and second-line TKIs to simulate the benefit of sequences after first-line ATEZO + BEVA. Therefore, the estimate of SORA and LENVA OS in RCTs may be overestimated compared to REGO, $\mathrm{CABO}$, and RAMU OS, given the use of different subsequent therapies in some patients after SORA or LENVA discontinuation. Fourth, the sequences including REGO as second-line therapy could have overestimated safety because RESORCE trial included only SORA-tolerant patients and SAEs may be higher in an unselected population [5]. Fifth, we were unable to calculate quality-adjusted life-years, since utilities were not available in the majority of the RCTs examined. Accordingly, we assessed LYG, and not quality-adjusted life-years, as the primary measure of efficacy. Sixth, we lacked data on potentially relevant effect modifiers such as microscopic vascular invasion, histological grading, gene profiling [38]. However, our meta-regression showed that BCLC stage C, ALBI grade 2, and AFP higher than $400 \mathrm{ng} / \mathrm{mL}$ were significantly associated with worse OS, confirming their prognostic usefulness. Seventh, the number of grade 3 and 4 SAEs was not separately reported in RCTs, hampering the evaluation of the degree and severity of SAEs in the calculation of ISER. Finally, due to the lack of individual data, our model cannot consider dropouts from first to second-line because of AEs or liver decompensation, which are well-demonstrated drivers of death in patients with HCC [39-42]. Therefore, time to liver decompensation [42] and time to treatment discontinuation due to AEs should be collected and reported in future trials. In conclusion, waiting for the results from RCTs and prospective real-world data evaluating firstline ICI-based combination followed by second-line mono- or combination TKI-based regimens [31], ATEZO + BEVA followed by LENVA or SORA could be considered optimal sequential options for advanced HCC.

\section{Conflict of Interest Statement}

Giuseppe Cabibbo participated in advisory board for Bayer, Eisai, and Ipsen. Maria Reig is a consultant at Bayer-Shering Pharma, BMS, Roche, Ipsen, AstraZeneca, Lilly, and BTG; attended paid conferences at Bayer-Shering Pharma, BMS, Gilead, and Lilly; received research grants from Bayer-Shering Pharma and Ipsen. Ciro Celsa received speaker fees from Eisai. Amit Singal served on advisory boards or as consultant for Genentech, Bayer, Eisai, Exelixis, Bristol Myers Squibb, and AstraZeneca. Jordi Bruix is a consultant at AbbVie, Adaptimmune, Arqule, Astra-Medimmune, Basilea, Bayer-Shering Pharma, Bio-Alliance, BMS, BTG-Biocompatibles, Eisai, Gilead, Incyte, Ipsen, Kowa, Lilly, MSD, Nerviano, Novartis, Polaris, Quirem, Roche, Sirtex, Sanofi, and Terumo; received research grants from Bayer and BTG; received educational grants from Bayer and BTG; attended paid conferences at Bayer, BTG, Astra-Zeneca, and Ipsen; gave paid talks at Bayer-Shering Pharma, BTG-Biocompatibles, Eisai, Terumo, Sirtex, and Ipsen. Calogero Cammà participated in the advisory board for Bayer, MSD/Merck, Ipsen, and Eisai. The other authors have no disclosure to declare.

\section{Funding Sources}

A.G.S. received Grant support from the National Institute of Health (NIH) R01 MD12565. J.B. received Grant support from Instituto de Salud Carlos III (PI18/00768), AECC (PI044031), and WCR (AICR) 16-0026. CIBERehd is funded by the Instituto de Salud Carlos III.

\section{Author Contributions}

All the authors take full responsibility for the study design, data analysis and interpretation, and preparation of the manuscript. All authors were involved in planning the analysis and drafting the manuscript. All authors approved the final draft of the manuscript.

\section{Data Availability Statement}

All data generated or analyzed during this study are included in this article and/or its online supplementary material files. Further inquiries can be directed to the corresponding author.

\section{Statement of Ethics}

The paper is exempt from Ethical Committee approval according to decision of Ethic Committee of Palermo and written consent was not required as only data extracted from published randomized controlled trials were used.

References

82
1 Bray F, Ferlay J, Soerjomataram I, Siegel RL, Torre LA, Jemal A. Global cancer statistics 2018: GLOBOCAN estimates of incidence and mortality worldwide for 36 cancers in 185 countries. CA Cancer J Clin. 2018;68:394424. [Erratum: CA Cancer J Clin. 2020;70: 313].

2 European Association for the Study of the Liver. EASL clinical practice guidelines: management of hepatocellular carcinoma. J Hepatol. 2018 Jul;69(1):182-236. 
3 Llovet JM, Ricci S, Mazzaferro V, Hilgard P, Gane E, Blanc JF, et al. Sorafenib in advanced hepatocellular carcinoma. N Engl J Med. 2008 Jul 24;359(4):378-90.

4 Finn RS, Qin S, Ikeda M, Galle PR, Ducreux M, Kim TY, et al. IMbrave150 investigators. Atezolizumab plus bevacizumab in unresectable hepatocellular carcinoma. N Engl J Med. 2020 May 14;382(20):1894-905.

5 Bruix J, Qin S, Merle P, Granito A, Huang YH, Bodoky $\mathrm{G}$, et al. Regorafenib for patients with hepatocellular carcinoma who progressed on sorafenib treatment (RESORCE): a randomised, double-blind, placebo-controlled, phase 3 trial. Lancet. 2017 Jan 7;389(10064): $56-66$.

6 Abou-Alfa GK, Meyer T, Cheng AL, ElKhoueiry AB, Rimassa L, Ryoo BY, et al. Cabozantinib in patients with advanced and progressing hepatocellular carcinoma. $\mathrm{N}$ Engl J Med. 2018 Jul 5;379(1):54-63.

7 Zhu AX, Kang YK, Yen CJ, Finn RS, Galle PR, Llovet JM, et al. REACH-2 study investigators. Ramucirumab after sorafenib in patients with advanced hepatocellular carcinoma and increased $a$-fetoprotein concentrations (REACH-2): a randomised, double-blind, placebo-controlled, phase 3 trial. Lancet Oncol. 2019 Feb;20(2):282-96.

8 Sonbol MB, Riaz IB, Naqvi SAA, Almquist DR, Mina S, Almasri J, et al. Systemic therapy and sequencing options in advanced hepatocellular carcinoma: a systematic review and network meta-analysis. JAMA Oncol. 2020 Dec 1;6(12):e204930.

9 Gordan JD, Kennedy EB, Abou-Alfa GK, Beg MS, Brower ST, Gade TP, et al. Systemic therapy for advanced hepatocellular carcinoma: ASCO guideline. J Clin Oncol. 2020 Dec 20; 38(36):4317-45.

10 Vogel A, Martinelli E, ESMO Guidelines Committee. Updated treatment recommendations for hepatocellular carcinoma (HCC) from the ESMO clinical practice guidelines. Ann Oncol. 2021 Jun;32(6):801-5.

11 Cheng AL, Kang YK, Chen Z, Tsao CJ, Qin S, Kim JS, et al. Efficacy and safety of sorafenib in patients in the Asia-Pacific region with advanced hepatocellular carcinoma: a phase III randomised, double-blind, placebo-controlled trial. Lancet Oncol. 2009 Jan;10(1): 25-34.

12 Johnson PJ, Qin S, Park JW, Poon RT, Raoul JL, Philip PA, et al. Brivanib versus sorafenib as first-line therapy in patients with unresectable, advanced hepatocellular carcinoma: results from the randomized phase III BRISKFL study. J Clin Oncol. 2013 Oct 1;31(28): 3517-24.

13 Cheng AL, Kang YK, Lin DY, Park JW, Kudo $M$, Qin S, et al. Sunitinib versus sorafenib in advanced hepatocellular cancer: results of a randomized phase III trial. J Clin Oncol. 2013 Nov 10;31(32):4067-75.
14 Cainap C, Qin S, Huang WT, Chung IJ, Pan $\mathrm{H}$, Cheng $\mathrm{Y}$, et al. Linifanib versus sorafenib in patients with advanced hepatocellular carcinoma: results of a randomized phase III trial. J Clin Oncol. 2015 Jan 10;33(2):172-9.

15 Zhu AX, Rosmorduc O, Evans TR, Ross PJ, Santoro A, Carrilho FJ, et al. SEARCH: a phase III, randomized, double-blind, placebo-controlled trial of sorafenib plus erlotinib in patients with advanced hepatocellular carcinoma. J Clin Oncol. 2015 Feb 20;33(6):55966.

16 Kudo M, Finn RS, Qin S, Han KH, Ikeda K, Piscaglia F, et al. Lenvatinib versus sorafenib in first-line treatment of patients with unresectable hepatocellular carcinoma: a randomised phase 3 non-inferiority trial. Lancet. 2018 Mar 24;391(10126):1163-73.

17 Kudo M, Finn RS, Morimoto M, Rau K-M, Ikeda M, Yen C-J, et al. Ramucirumab for patients with intermediate-stage hepatocellular carcinoma and elevated alpha-fetoprotein: pooled results from two phase 3 studies (REACH and REACH-2). Liver Cancer. 2021; 10(5):451-60.

18 Zhu AX, Park JO, Ryoo BY, Yen CJ, Poon R, Pastorelli D, et al. Ramucirumab versus placebo as second-line treatment in patients with advanced hepatocellular carcinoma following first-line therapy with sorafenib (REACH): a randomised, double-blind, multicentre, phase 3 trial. Lancet Oncol. 2015 Jul;16(7): 859-70.

19 Vogel A, Frenette C, Sung M, Daniele B, Baron A, Chan S L, et al. Baseline liver function and subsequent outcomes in the phase $3 \mathrm{RE}$ FLECT study of patients with unresectable hepatocellular carcinoma. Liver Cancer. 2021;10(5):510-21.

20 Miksad R, Cicin I, Chen Y, Klumpen H, Kim $\mathrm{S}$, Lin Z, et al. O-022outcomes based on albumin-bilirubin (ALBI) grade in the phase 3 CELESTIAL trial of cabozantinib versus placebo in patients with advanced hepatocellular carcinoma (HCC). Ann Oncol. 2019;30.

21 Kudo M, Galle PR, Brandi G, Kang YK, Yen CJ, Finn RS, et al. Effect of ramucirumab on ALBI grade in patients with advanced HCC: results from REACH and REACH-2. JHEP Rep. 2021 Nov 13;3(2):100215

22 Cabibbo G, Celsa C, Enea M, Battaglia S, Rizzo GEM, Busacca A, et al. Progression-free survival early assessment is a robust surrogate endpoint of overall survival in immunotherapy trials of hepatocellular carcinoma. Cancers. 2020 Dec 30;13(1):90.

23 Guyot P, Ades AE, Ouwens MJ, Welton NJ. Enhanced secondary analysis of survival data: reconstructing the data from published $\mathrm{Ka}$ plan-Meier survival curves. BMC Med Res Methodol. 2012 Feb 1;12:9.

24 Schnipper LE, Davidson NE, Wollins DS, Tyne C, Blayney DW, Blum D, et al. American Society of Clinical Oncology statement: a conceptual framework to assess the value of cancer treatment options. J Clin Oncol. 2015 Aug 10;33(23):2563-77.
25 Bruix J, Chan SL, Galle PR, Rimassa L, Sangro B. Systemic treatment of hepatocellular carcinoma: an EASL position paper. J Hepatol. 2021 Oct;75(4):960-74.

26 NCT03713593. Available from: https://clinicaltrials.gov/ct2/show/NCT03713593 Accessed 2021 Apr 13.

27 Iavarone M, Cabibbo G, Piscaglia F, Zavaglia C, Grieco A, Villa E, et al. SOFIA (SOraFenib Italian assessment) study group. Field-practice study of sorafenib therapy for hepatocellular carcinoma: a prospective multicenter study in Italy. Hepatology. 2011 Dec;54(6): 2055-63.

28 Cammà C, Cabibbo G, Petta $\mathrm{S}$, Enea M, Iavarone $\mathrm{M}$, Grieco A, et al. Cost-effectiveness of sorafenib treatment in field practice for patients with hepatocellular carcinoma. Hepatology. 2013 Mar;57(3):1046-54.

29 Reiss KA, Yu S, Mamtani R, Mehta R, D'Addeo K, Wileyto EP, et al. Starting dose of sorafenib for the treatment of hepatocellular carcinoma: a retrospective, multi-institutional study. J Clin Oncol. 2017 Nov 1;35(31): 3575-81.

30 Yoo C, Kim JH, Ryu MH, Park SR, Lee D, Kim $\mathrm{KM}$, et al. Clinical outcomes with multikinase inhibitors after progression on first-line atezolizumab plus bevacizumab in patients with advanced hepatocellular carcinoma: a multinational multicenter retrospective study. Liver Cancer. 2021 Apr;10(2):107-14.

31 NCT04770896. Available from: https://clinicaltrials.gov/ct2/show/study/NCT04770896. Accessed 2021 Mar 24.

32 Finn RS, Merle P, Granito A, Huang YH, Bodoky G, Pracht M, et al. Outcomes of sequential treatment with sorafenib followed by regorafenib for HCC: additional analyses from the phase III RESORCE trial. J Hepatol. 2018 Aug;69(2):353-8.

33 Kelley RK, Ryoo BY, Merle P, Park JW, Bolondi L, Chan SL, et al. Second-line cabozantinib after sorafenib treatment for advanced hepatocellular carcinoma: a subgroup analysis of the phase 3 CELESTIAL trial. ESMO Open. 2020 Aug;5(4):e000714.

34 Celsa C, Cabibbo G, Enea M, Battaglia S, Rizzo GEM, Busacca A, et al. Are radiological endpoints surrogate outcomes of overall survival in hepatocellular carcinoma treated with transarterial chemoembolization? Liver Int. 2021 May;41(5):1105-16.

35 Reig M, Rimola J, Torres F, Darnell A, Rodriguez-Lope C, Forner A, et al. Postprogression survival of patients with advanced hepatocellular carcinoma: rationale for second-line trial design. Hepatology. 2013 Dec;58(6):202331.

36 Hsu C, Lin EP, Shyr Y. Development and evaluation of a method to correct misinterpretation of clinical trial results with long-term survival. JAMA Oncol. 2021;7(7):1041-44.
Sequential Systemic Treatments for

Advanced Hepatocellular Carcinoma
Liver Cancer 2022;11:75-84

DOI: $10.1159 / 000520278$ 
37 Stinnett AA, Mullahy J. Net health benefits: a new framework for the analysis of uncertainty in cost-effectiveness analysis. Med Decis Making. 1998 Apr-Jun;18(2 Suppl):S68-80.

38 Villa E, Critelli R, Lei B, Marzocchi G, Cammà C, Giannelli G, et al. Neoangiogenesis-related genes are hallmarks of fast-growing hepatocellular carcinomas and worst survival. Results from a prospective study. Gut. 2016 May; 65(5):861-9.
39 Cabibbo G, Enea M, Attanasio M, Bruix J, Craxì $\mathrm{A}, \mathrm{Cammà} \mathrm{C}$. A meta-analysis of survival rates of untreated patients in randomized clinical trials of hepatocellular carcinoma. Hepatology. 2010 Apr;51(4):1274-83.

40 Cabibbo G, Petta S, Barbara M, Attardo S, Bucci L, Farinati F, et al. Hepatic decompensation is the major driver of death in HCVinfected cirrhotic patients with successfully treated early hepatocellular carcinoma. J Hepatol. 2017 Jul;67(1):65-71.
41 Cabibbo G, Celsa C, Calvaruso V, Petta S, Cacciola I, Cannavò MR, et al. Direct-acting antivirals after successful treatment of early hepatocellular carcinoma improve survival in HCV-cirrhotic patients. J Hepatol. 2019 Aug; 71(2):265-73.

42 Reig M, Cabibbo G. Antiviral therapy in the palliative setting of HCC (BCLC B and C). J Hepatol. 2021;74(5):1225-33. 